\title{
Frequency of Various Dermatoses Requiring Histopathological Evaluation For Definite Diagnosis; A Retrospective Analysis
}

\section{Hira Tariq, ${ }^{1}$ Rabia Mukhtar, ${ }^{2}$ Abeer Fahad, ${ }^{3}$ Tariq Rashid, ${ }^{4}$ Ambereen Anwar, ${ }^{5}$ Ameena Ashraf ${ }^{6}$}

\begin{abstract}
Objective: To assess the frequency of various dermatological disorders those require histopathological analysis for definite diagnosis.

Methods: We included 669 patients of either gender and all ages in this retrospective study, carried out in the Department of Dermatology, Jinnah Hospital, Lahore for a duration of 2 years. Their clinical data and histopathological reports were analyzed. The dermatoses were categorized into (a) papulosquamous disorders, (b) bullous disorders, (c) eczemas, (d) neoplasia, (e) granulomatous disorders, (f) connective tissue diseases, (g) drug reactions, (h) vasculitides, (i) chronic ulcers and (j) miscellaneous disorders.

Results: The frequency of various dermatoses noted were as follows: papulosquamous disorders $28.25 \%$, bullous disorders $11.5 \%$, granulomatous disorders $11.5 \%$, connective tissue diseases $10.61 \%$, miscellaneous disorders $10 \%$, eczemas $9.4 \%$, chronic ulcers 7.17 neoplasia $6.8 \%$, vasculitides $2.84 \%$ and drug reactions were $1.79 \%$ of total biopsies.

Conclusion: The frequency of different diagnostic groups was unique in some respects and confirmed to other studies in others. The significantly high frequency of papulosquamous disorders highlighted the importance of these disorders.

Key words: Skin biopsy, papulosquamous disorders, bullous disorders, eczemas, neoplasia, granulomatous disorders, connective tissue diseases, drug reactions, vasculitides, chronic ulcers.
\end{abstract}

\section{Introduction}

$\mathrm{O}$ btaining a definite diagnosis is of utmost importance in skin disorders since management depends on it. Skin biopsy is useful tool for improving diagnostic accuracy in majority of dermatoses. ${ }^{1}$ Interpretation of histopathology requires provision of adequate clinical information. Skin disorders constitute a major proportion of outdoor and indoor patients in developing countries. ${ }^{2}$ Diseases of skin are a source of significant physical, social and psychological disability. No age is immune to these disorders. Clinical consequences vary from troublesome itching to death. ${ }^{3}$ The prevalence of these diseases is often

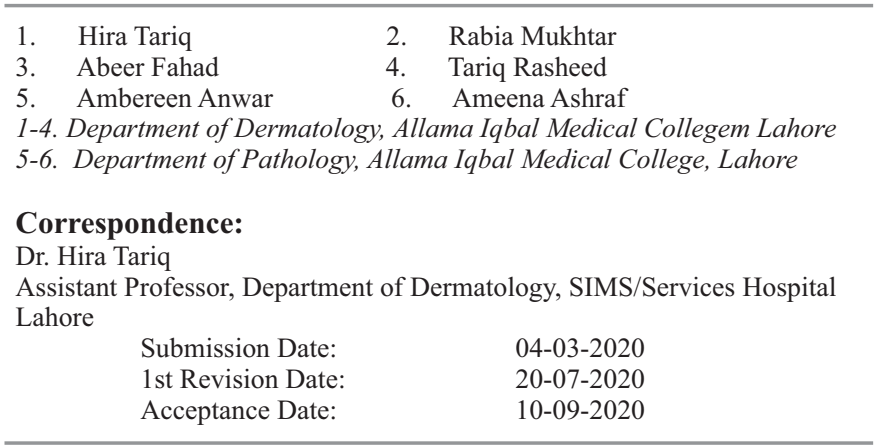

underestimated. Their distribution varies in different parts of the world and even within the same country. ${ }^{4}$ Assessment of their frequency is important as many of these diseases are preventable. ${ }^{5,6}$ Therefore, it is important to know the spectrum of disorders that require skin biopsy as adjunct to clinical diagnosis. In this study, we aim to analyse the pattern of dermatoses requiring skin biopsies. It will also help the concerned personnel to set their priorities.

\section{Methods}

Retrospective analysis of histopathology reports of 669 patients was conducted at the Department of Dermatology, Jinnah Hospital, Lahore. These patients underwent biopsies from 1st April 2017 to 31 st March 2019. Patients of either gender and all ages were included. Based on the histopathological diagnoses biopsies were divided into following groups: (a) papulosquamous disorders, (b) bullous disorders, (c) eczemas, (d) neoplasia, (e) granulomatous disorders, (f) connective tissue diseases, (g) drug reactions, (h) vasculitides, (i) chronic ulcers and (j) miscellaneous disorders. 
Disorders like lichen planus, psoriasis and pityriasis lichenoides chronica were lumped together as papulosquamous disorders. Category of blistering diseases included pemphigus vulgaris, pemphigus foliaceous, bullous pemphigoid, linear IgA disease, dermatitis herpetiformis, phorphyria and epidermolysis bullosa. Connective tissue diseases included lupus erythematosus, dermatomyositis, morphea and mixed connective tissue disease. Granulomatous skin diseases included tuberculosis, fungal infections, leishmaniasis and sarcoidosis. Neoplastic diseases encountered were squamous cell carcinoma, basal cell carcinoma, melanoma and mycosis fungoides. Chronic ulcers included pyoderma gangrenosum, arterial and venous ulcers. Other less common diseases like hidradenitis suppurativa, keloid, sebaceous hyperplasia were put in the miscellaneous group. Results were tabulated and analyzed using percentage frequencies.

\section{Results}

Frequency of various disorders were analysed using SPSS 25. Table 1 represents the frequencies of various disease groups. The most common group of dermatoses was papulosquamous disorders with a frequency of $28.25 \%$. Second most frequent skin disease groups were granulomatous and blistering disorders, each accounting for $11.5 \%$ of all biopsies. Connective tissue diseases and eczemas were responsible for $10.6 \%$ and $9.4 \%$ of biopsies respectively. Chronic ulcers were noted at a frequency of $7.17 \%$. Neoplastic lesions constituted $6.8 \%$ of biopsies. Vasculitides constituted $2.84 \%$, drug reactions $1.79 \%$ and miscellaneous disorders were $10 \%$ of the total biopsies.

\section{Fig 1: Relative frequencies of various dermatoses}

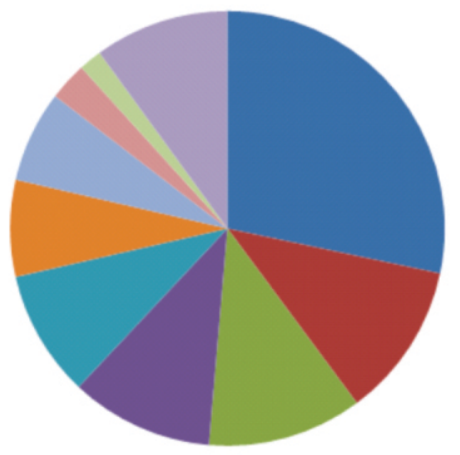

- Papulosquamous disorders

- Blistering disorders

= Granulomatous disorders

n Connective tissue diseases

- Eczemas

" Chronic ulcers

neoplasia

= Vascilitides

= Drug reactions

niscellaneous
Table 1: Frequency of Different Pathological Groups $(n=669)$

\begin{tabular}{lc}
\hline \multicolumn{1}{c}{ Types of lesions } & N (\%) \\
\hline Papulosquamous disorders & $189(28.25)$ \\
Blistering disorders & $77(11.5)$ \\
Granulomatous disorders & $77(11.5)$ \\
Connective tissue diseases & $71(10.61)$ \\
Eczemas & $63(9.4)$ \\
Chronic ulcers & $48(7.17)$ \\
Neoplastic lesions & $46(6.8)$ \\
Vascilitides & $19(2.84)$ \\
Drug reactions & $12(1.7)$ \\
Miscellaneous lesions & $67(10)$ \\
\hline
\end{tabular}

\section{Discussion}

Cutaneous disorders may arise from skin or may be manifestation of systemic disorders. ${ }^{7}$ In clinical dermatology, we come across a variety of disorders that mimic each other immensely. Similarly, histopathological features of various disorders too are so nonspecific and overlapping that making a histopathological diagnosis is mostly impossible without adequate clinical data. ${ }^{8}$

In our study, among various categories of disorders, papulosquamous disorders formed the majority of dermatoses that underwent biopsy. This corresponds to the findings of other studies. ${ }^{9,10}$ Papulosquamous disorders are a wide group of skin diseases and are very commonly confronted by the histopathologist. The next common groups were granulomatous and blistering disorders which occurred with equal frequency of $11.5 \%$. This is contradictory to some other studies which reported infectious diseases to be second commonest.

This is probably because of advanced medical management of these orders which led to reduced burden and hence less requirement for them to be biopsied. Connective tissue diseases comprised the next commonest group with frequency of $10.61 \%$, however, due to lack of advanced modalities of diagnosis like immunohistochemistry and immunofluorescence, few of the diagnoses could not be confirmed. Miscellaneous group comprised 10\% of biopsies and included a multitude of disorders that couldn't be included in other groups mentioned. Eczemas constituted the next frequent group with 
frequency of $9.4 \%$. Chronic ulcers comprised $7.17 \%$ of the biopsies and were caused due to arterial, venous or mixed pathology. Pyoderma gangrenosum was another common cause. Neoplasia constituted $6.8 \%$ of biopsies which is quite alarming as our patients come from an area with a high level of exposure to sunlight. ${ }^{13}$ This further highlights the role of sunlight in these diseases. ${ }^{14}$

The major limitation was lack of advanced modalities of diagnosis like immunohistochemistry and immunofluorescence to confirm histopathological diagnoses.

\section{Author's Contribution \\ HT: Main Author \\ RM: Co-Author \\ AF: Collection of Cases \\ TR: Supervisor and HOD \\ AA, AA: Histopathology Analysis}

\section{References}

1. Chrysovalantis K, Stamatis G, Christina A, Andreas D, Dimitris R, "Skin Biopsy in the Context of Dermatological Diagnosis: A Retrospective Cohort Study," Dermatology Research and Practice, vol. 2014, Article ID 734906, 2014.

2. Bangash E, Iqbal T, Iqbal S. Frequency of skin diseases presenting to a Primary Health Care Center in rural Islamabad, Pakistan. Rawal Med J. 2014;39: 403-5.

3. Karimkhani C, Dellavalle RP, Coffeng LE, Flohr C, Hay RJ, Langan SM et al. Global skin disease morbidity and mortality: an update from the global burden of disease study 2013. JAMA Dermatol. 2017;153:406- 12.

4. Dalgard F, Svensson A, Holm J0, Sundby J. Self- reported skin morbidity among adults: associations with quality of life and general health in a Norwegian survey. J Investig Dermatol Symp Proc. 2004;9:1205.

5. Eisner P. Skin protection in the prevention of skin diseases. Curr Probl Dermatol. 2007;34:1-10.

6. Diepgen TL. Occupational skin diseases. J Dtsch Dermatol Ges. 2012;10:297-313. doi:10.111 1/j.16100387.2012.07890.

7. Cahill J, Sinclair R. Cutaneous manifestations of systemic disease. Aust Fam Physician. 2005;34:33540.

8. Aslan C, Gotkay F, Mansur AT, Aydingoz IE, Gunes P, Ekmekci TR. Clinicopathological consistency in skin disorders: A retrospective study of 3949 pathological reports. J Am Acad Dermatol. 2012;66:393400.

9. Kumar A, Shrestha PR, Pun J, Thapa P, Manandhar M, Sathian B. Profile of skin biopsies and pattern of skin cancer in a tertiary care center of Western Nepal. Asian Pac J Cancer Prev. 2015; 16:3403-6.

10. Goltz RW. Dermatitis, papulosquamous disorders and psoriasis. Pathology. 1981;13:389.

11. Din T, Butt AQ, Bangash FA, Abbas H. Burden of skin diseases at a tertiary care hospital. J Rawal Med Coll. 2010;14:90-2.

12. Abdel-Hafez K, Abdel-Aty MA, Hofny ERM. Prevalence of skin diseases in rural areas of Assiut Governorate, Upper Egypt. Int J Dermatol. 2003;42: 887-92.

13. Humayun Q, Iqbal R, Azam I, Khan AH, Siddiqui AR, Ansari NB. Development and validation of sunlight exposure measurement questionnaire (SEM-Q) for use in adult population residing in Pakistan. BMC Public Health 2012;12:421.

14. Ananthaswamy HN. Sunlight and skin cancer. J 